\title{
DEDDNTE

\section{5) Ressonância magnética funcional em idosos: cognição e deglutição}

Lucia Figueiredo Mourão ${ }^{1}$

\section{Resumo}

O envelhecimento cerebral ocorre por diferentes fatores biológicos e funcionais. O declínio funcional depende do quanto o cérebro é requerido ao longo da vida: quanto mais atividades intelectuais foram realizadas, mais tempo o cérebro demorará para perder suas conexões. Além disso, observam-se alterações cognitivas que impactam também na deglutição, na redução de sensibilidade intra-oral e na diminuição da percepção gustativa. Os estudos estão avançando com o uso da ressonância magnética funcional em idosos.

Palavras-chave: Ressonância magnética funcional. Cognição. Deglutição.

Inevitavelmente, o envelhecimento cerebral ocorre, porém dependerá de diferentes fatores biológicos e funcionais. Do ponto de vista biológico, são observados diversos processos de envelhecimento cerebral: atrofia cerebral com dilatação de sulcos e ventrículos; perda de neurônios; degeneração granulovacuolar; presença de placas neuríticas; formação de corpos de Lewy a partir a da alfa sinucleína (NORDON, 2009). No entanto, o declinio funcional vai depender do quanto o cerebro é requerido ao longo da vida, ou seja, quanto mais atividades intelectuais foram realizadas, mais tempo o cerebro demorará para perder suas conexões e, consequentemente, apresentar uma perda sintomática, tendo em vista sua excepcional capacidade plástica (NORDON, 2009). Assim, as alterações características do envelhecimento levam aos déficits cognitivos comumente observados como naturais no envelhecimento: esquecimento de fatos recentes, dificuldades de cálculo, alterações de atenção pronunciada.

Além disso, com o envelhecimento observam-se alterações cognitivas que impactam na preparação, reconhecimento e planejamento motor associadas às alterações no controle sensório-motor, que levam ao aumento de tempo de trânsito oral, associada a diminuição de força e pressão da língua, prejuízo da manipulação e ejeção do bolo alimentar, redução de sensibi-

Professora no Programa de Pós-Graduação em Gerontologia da Universidade Estadual de Campinas, Faculdade de Ciências Médicas. Endereço para correspondência: Rua Tessália Vieira de Camargo, 126. CEP: 13083-887 - Campinas, SP, Brasil. Email: lumourao@fcm.unicamp.br 
lidade intra-oral, diminuição da percepção gustativa e aumento da incidência de penetrações (JEAN, 2001; MILLER, 1986; MALANDRAKI et al, 2011).

Estudos com neuroimagem mostraram que, além das áreas corticais distintas do tronco cerebral (córtex sensório-motor primário), as áreas de integração sensório-motora, insula, córtex cingulado anterior e a área motora suplementar adjacente, estão significativamente envolvidas no processamento da deglutição (MALANDRAKI et al, 2011; 2010; MARTIN et al, 2007). Estudos realizados na população idosa identificaram a ativação neuronal durante a deglutição de saliva e de água maior ativação nas regiões corticais do que subcorticais, com destaque para o córtex cingulado, giro pós-central, com maior lateralização para o hemisfério esquerdo para ambas as tarefas e maior ativação do hemisfério direito, no córtex pré-motor e pré-frontal para a tarefa de deglutição de água, o que sugere uma ação compensatória na deglutição resultante do declínio sensório-motor associado a idade (MALANDRAKI et al, 2010).

Do ponto de vista clínico, as alterações relacionadas ao processo de deglutição podem ser identificadas por algumas ferramentas de triagem, como o Eating Assessment Tool (EAT-10) (ROFES et al, 2014). O EAT-10 é um questionário de triagem da deglutição auto-aplicável, considerado uma ferramenta relevante para a identificação inicial do risco de disfagia e aspiração, favorecendo a indicação de intervenção multidisciplinar o mais precoce possível. $\mathrm{O}$ instrumento tem alta sensibilidade em torno de $80 \%$ e especificidade variando de 70 à $75 \%$ (ROFES et al, 2014; CHENEY et al, 2015).

Desse modo, acredita-se que entender a conectividade cerebral e o padrão de deglutição em idosos saudáveis, é primordial para que sejam estabelecidas medidas de prevenção ou estratégias de reabilitação, a fim de evitar maiores dificuldades do ponto de vista da deglutição, assim como outros agravos como a broncoaspiração e a pneumonia aspirativa.

\title{
Functional magnetic resonance imaging in the elderly: cognition and swallowing
}

\begin{abstract}
Cerebral aging occurs due to different biological and functional factors. Functional decline depends on how much the brain is required throughout life: the more intellectual activities have been performed, the longer the brain will take to lose its connections. In addition, we observe cognitive alterations that also affect swallowing, reduction of intra-oral sensitivity and reduction of gustatory perception. Studies are advancing with the use of functional magnetic resonance imaging in the elderly.
\end{abstract}

Keywords: Functional magnetic resonance imaging. Cognition. Swallowing.

\section{Referências}

CHENEY, D.M. et.al. The ability of the 10-Item Eating Assessment Tool (EAT-10) to Predict Aspiration Risk in Persons with Dysphagia. Ann Otol Rhinol Laryngol. 2015;124(5):351-4.

JEAN, A. Brain stem control of swallowing: Neuronal network and cellular mechanisms. Physiological Reviews. $2001 ; 81(2): 929-960$.

MALANDRAKI, G.A.; SUTTON, B.P.; PERLMAN, A.L.; KARAMPINOS, D.C. Age-related differences in laterality of cortical activations in swallowing. Dysphagia. 2010 Sep;25(3):238-49. 
MALANDRAKI, G.A.; SUTTON, B.P.; PERLMAN, A.L.; KARAMPINOS, D.C. Reduced Somatosensory Activations in Swallowing With Age. Human Brain Mapping. 2011; 32:730-743

MARTIN, R.E.; BARR, A.; MACINTOSH, B.; SMITH, R.; STEVENS, T.K.; TAVES, D.; GATI, J.; MENON, R.; HACHINSKI, V. Cerebral cortical processing of swallowing in older adults. Exp Brain Res. 2007; 176:12-22.

MILLER, A. J. Neurophysiological basis of swallowing. Dysphagia. 1986: 1;91-100.

NORDON, D.G.; GUIMARÃES, R.R.; KOZONOE, D.Y.; MANCILHA, V.S.; NETO, V.S.D. Perda cognitiva em idosos. Rev Fac Ciêne Méd, Sorocaba 2009;11(3):5-8

ROFES, L.; ARREOLA, V.; MUKHERJEE, R.; CLAVÉ, P. Sensivity and Specificity of the Eating Assessment Tool and the Volume-Viscosity Swallow Test for Clinical Evaluation of Oropharyngeal Dysphagia. Neurogastroenterol Mot. 2014; 26: 1256-1265. 